\title{
R-function theory method for free vibration of slip clamped trapezoidal shallow spherical shell on Winkler foundation
}

\author{
Shanqing $\mathrm{Li}^{1, \mathrm{a}}$ \\ ${ }^{1}$ MOE Key Laboratory of Disaster Forecast and Control in Engineering, Institute of Applied \\ Mechanics, Jinan University, Guangzhou 510632, China
}

alishanqing09@163.com

\begin{abstract}
Keywords: R-function, Green's function, integral equation, Winkler foundation, free vibration, shallow spherical shell

Abstract. In this paper, the R-function theory(RFT) is applied to solve the free vibration of slip clamped trapezoidal shallow spherical shell on Winkler foundation. Firstly the fundamental solution of the biharmonic operator, the boundary equation and the R-function are used to construct the quasi-Green's function. Then the model governing differential equation of the problem is reduced to the Fredholm integral equation of the second kind by Green's formula. The singularity of the kernel of the integral equation is overcome by choosing a suitable form of the normalized boundary equation by the R-function. A numerical example shows that this method is an effective numerical method.
\end{abstract}

\section{Introduction}

In the paper, the R-function theory proposed by Rvachev[1] are utilized. The free vibration of slip clamped trapezoidal shallow spherical shell on Winkler foundation is studied. A quasi-Green function is established by using the fundamental solution and the boundary equation of the problem. This function satisfies the homogeneous boundary condition of the problem, but it does not satisfy the fundamental differential equation. The key point of establishing the quasi-Green function consists in describing the boundary of the problem by normalized equation $\omega=0$ and the domain of the problem by inequality $\omega>0$. There are multiple choices for the normalized boundary equation. Based on a suitably chosen form of the normalized boundary equation, a new normalized boundary equation can be established such that the singularity of the kernel of the integral equation is overcome. For any complicated area, a normalized boundary equation can always be found according to the R-function theory. Thus, the problem can always be reduced to the Fredholm integral equation of the second kind without singularity. Using the present method, Li and Yuan solved successfully the free vibration of clamped thin plates[2], the simply-supported thin plate[3,4] and shallow spherical shells[5,6]. For the first time, the proposed R-function theory method is applied to study the free vibration problem of slip clamped trapezoidal shallow spherical shell on Winkler foundation. The numerical example demonstrates the efficiency and the feasibility of the R-function theory method.

\section{Fundamental equations}

The governing differential equations of the free problem of slip clamped trapezoidal shallow spherical shell on Winkler foundation [7] can be expressed as follow

$$
\nabla^{4} \varphi(\mathbf{x}, t)-\frac{E h}{R} \nabla^{2} w(\mathbf{x}, t)=0, \quad \mathbf{x} \in \Omega,
$$

and $D \nabla^{4} w(\mathbf{x}, t)+\frac{1}{R} \nabla^{2} \varphi(\mathbf{x}, t)+k w(\mathbf{x}, t)+\bar{m} \frac{\partial^{2} w(\mathbf{x}, t)}{\partial t^{2}}=0, \quad \mathbf{x} \in \Omega$,

where $\nabla^{4}=\left(\partial^{2} / \partial x_{1}^{2}+\partial^{2} / \partial x_{2}^{2}\right)^{2}$ is the biharmonic operator, $\varphi$ is the stress function, $w$ is the radial deflection of the shell, $R$ is the radius of curvature of the shell, $\mathbf{x}=\left(x_{1}, x_{2}\right), \Omega$ is the domain of the trapezoid of shallow spherical shells in Cartesian coordinates, $t$ is time; and $D=E h^{3} /\left(12\left(1-v^{2}\right)\right)$ is the flexural rigidity of the shell, in which $h$ is the thickness of the shell, and $E$ and $v$ are Young's modulus and Poisson's ratio, respectively. 
The slip clamped boundary conditions can be written as

$$
\left.w\right|_{\Gamma}=\left.\frac{\partial w}{\partial n}\right|_{\Gamma}=\left.\varphi\right|_{\Gamma}=\left.\nabla^{2} \varphi\right|_{\Gamma}=0
$$

where $\nabla^{2}=\partial^{2} / \partial x_{1}^{2}+\partial^{2} / \partial x_{2}^{2}$ is the Laplace operator, and $\Gamma=\partial \Omega$ is the boundary of the domain $\Omega$.

Making use of Eqs.(1) and (3), we can easily obtain

$\nabla^{2} \varphi=w E h / R$.

Substituting Eq.(4) into Eq.(2) yields

$$
D \nabla^{4} w+\frac{E h}{R^{2}} w+k w+\bar{m} \frac{\partial^{2} w}{\partial t^{2}}=0 .
$$

The model governing differential equation can be obtained as follow

$$
\begin{aligned}
& D \nabla^{4} W(\mathbf{x})+\frac{E h}{R^{2}} W(\mathbf{x})+k W(\mathbf{x})-\bar{\omega}^{2} \bar{m} W(\mathbf{x})=0, \quad \mathbf{x} \in \Omega \\
& W=0, \quad \frac{\partial W}{\partial n}=0, \quad \mathbf{x} \in \Gamma .
\end{aligned}
$$

where $W$ is the model function.

\section{Integral equations}

Let $\omega=0$ be the normalized boundary equation of the first-order on the boundary $\Gamma$, i.e.[1]

$$
\omega(\mathbf{x})=0,|\nabla \omega|=1, \quad \mathbf{x} \in \Gamma \text { and } \omega(\mathbf{x})>0, \quad \mathbf{x} \in \Omega
$$

The quasi-Green function can be established as follows:

$$
\begin{gathered}
G(\mathbf{x}, \xi)=-r^{2} \ln r-e(\mathbf{x}, \xi), \\
\text { and } e(\mathbf{x}, \xi)=-r^{2} \ln R_{1}+2 \omega(\mathbf{x}) \omega(\xi),
\end{gathered}
$$

where $r=\|\xi-\mathbf{x}\|=\sqrt{\left(\xi_{1}-x_{1}\right)^{2}+\left(\xi_{2}-x_{2}\right)^{2}}$ and $R_{1}=\sqrt{r^{2}+4 \omega(\xi) \omega(\mathbf{x})}$,

in which $\mathbf{x}=\left(x_{1}, x_{2}\right)$ and $\xi=\left(\xi_{1}, \xi_{2}\right)$. Obviously, the quasi-Green function $G(\mathbf{x}, \xi)$ satisfies the following condition

$$
\left.G(\mathbf{x}, \xi)\right|_{\xi \in \partial \Omega}=0 \text { and }\left.\frac{\partial G(\mathbf{x}, \xi)}{\partial n}\right|_{\xi \in \partial \Omega}=0 \text {. }
$$

In order to reduce the boundary value problems Eq.(7) and Eq.(8) into an integral equation, the following Green's formula of sets of function $C^{4}(\Omega)$, i.e., $U, V \in C^{4}(\Omega \cup \Gamma)$, is applied

$$
\int_{\Omega}\left(V \nabla^{4} U-U \nabla^{4} V\right) d_{\xi} \Omega=\int_{\partial \Omega}\left[V \frac{\partial}{\partial n}\left(\nabla^{2} U\right)-\frac{\partial V}{\partial n} \nabla^{2} U-U \frac{\partial}{\partial n}\left(\nabla^{2} V\right)+\frac{\partial U}{\partial n} \nabla^{2} V\right] d_{\xi} \Gamma .
$$

Replacing $U$ and $V$ in Eq.(10) by $w$ and Green quasifunction $G$, noticing that $(1 / 8 \pi) r^{2} \ln r$ is the fundamental solution[6] of the biharmonic operator, and using Eqs. (7), (8) and (13), we can easily obtain

$$
W(\mathbf{x})=\int_{\Omega} W(\xi) K(\mathbf{x}, \xi) d_{\xi} \Omega,
$$

where $K(\mathbf{x}, \boldsymbol{\xi})=-\frac{1}{8 \pi}\left(\frac{\partial^{4}}{\partial \xi_{1}^{4}}+2 \frac{\partial^{4}}{\partial \xi_{1}^{2} \partial \xi_{2}^{2}}+\frac{\partial^{4}}{\partial \xi_{2}^{4}}\right) q(\mathbf{x}, \boldsymbol{\xi})+\frac{1}{8 \pi}\left(\frac{E h}{D R^{2}}+\frac{k}{D}-\frac{\bar{\omega}^{2} \overline{\mathrm{m}}}{\mathrm{D}}\right) \mathrm{G}(\mathbf{x}, \boldsymbol{\xi})$. 
Substituting Eq.(11) into Eq.(16), we can obtain the expression of $K(\mathbf{x}, \boldsymbol{\xi})$ in detail.

$K(\mathbf{x}, \xi)$ in Eq.(16) appears discontinuous only if $R_{1}=0$, i.e., both $\mathbf{x}=\boldsymbol{\xi}$ and $\omega=0$ come into existence. Actually, when $\mathbf{x}=\xi$, Eq.(16) can be reduced to

$$
\left.K(\mathbf{x}, \xi)\right|_{\mathbf{x}=\xi}=\frac{1+\omega \nabla^{2} \omega-(\nabla \omega)^{2}}{\pi \omega^{2}}-\frac{1}{4 \pi} \omega \nabla^{4} \omega-\frac{1}{4 \pi}\left(\frac{E h}{D R^{2}}+\frac{k}{D}-\frac{\bar{\omega}^{2} \overline{\mathrm{m}}}{\mathrm{D}}\right) \omega^{2}
$$

To make the kernel of the integral equation $K(\mathbf{x}, \xi) \in C(\Omega \cup \partial \Omega)$, A normalized boundary equation will be constructed to ensure the continuity of $K(\mathbf{x}, \xi)$ in the following. It can be easily testified that

$$
\omega=\left[3 \omega_{0}+\omega_{0}^{2} \nabla^{2} \omega_{0}-\omega_{0}\left(\nabla \omega_{0}\right)^{2}\right] / 2
$$

where $\omega_{0}=0$ is the normalized equation of the boundary $\Gamma$, i.e., $\omega_{0}$ satisfies Eq.(9). Obviously, equation $\omega$ is also a normalized boundary equation of the first-order.

Based on a suitably chosen normalized boundary equation $\omega_{0}=0$, a new normalized boundary equation $\omega=0$ can be constructed by using Eq.(18), which ensure the continuity of the integral kernel $K(\mathbf{x}, \xi)$ everywhere in the integral domain.

In order to obtain numerical results of the boundary problem, the integral equation (15) can be discretized into the homogeneous linear algebraic equation. The determinant of the matrix of coefficients of homogeneous linear algebraic equations Eq.(15) must be equal to zero. So, the natural frequency $\bar{\omega}$ or $f=\bar{\omega} /(2 \pi)$ can be obtained by solving the equation.

\section{Numerical example}

A slip clamped trapezoidal shallow spherical shell is shown in Fig.1, and we set $a=e=1.2$, $b=d=1.0$, and $c=1.1$. The following reference parameters are used: the radius of curvature of the shell $R=3$, the thickness of the shell $h=0.1$, Poisson's ratio $v=0.3$, Young's modulus $E=3 \times 10^{9}$, the elastic foundation coefficient $k=2 \times 10^{7}$, and mass density per unit area $\bar{m}=780$. According to the theory of R-function[1], a normalized boundary equation of the first rank $\omega_{0}=0$ can be constructed from the following equation:

$$
\omega_{0}=\omega_{1}+\omega_{2}+\omega_{3}-\sqrt{\omega_{1}^{2}+\omega_{2}^{2}}-\sqrt{\omega_{1}^{2}+\omega_{3}^{2}}-\sqrt{\omega_{2}^{2}+\omega_{3}^{2}}+\sqrt{\omega_{1}^{2}+\omega_{2}^{2}+\omega_{3}^{2}}
$$

where $\omega_{2}=\left(\frac{a c}{a-b}+\frac{c}{a-b} x_{1}-x_{2}\right) / \sqrt{1+\left(\frac{c}{a-b}\right)^{2}} \geq 0, \omega_{3}=\left(\frac{a c}{a-b}-\frac{c}{a-b} x_{1}-x_{2}\right) / \sqrt{1+\left(\frac{c}{a-b}\right)^{2}} \geq 0$, and $\omega_{1}=\left(c-x_{2}\right) x_{2} / c \cdot \omega_{1}=0, \omega_{2}=0$ and $\omega_{3}=0$ denote various parts of the boundary of trapezoidal shallow spherical shell, respectively. Numerical results by the R-function theory(RFT) and ANSYS finite-element method (FEM) are shown in Table 1 for comparison. Numerical results by $11 \times 11$ trapezoidal integral domain of the R-function theory(RFT) show fine agreement with the FEM solution by $200 \times 200$. It shows the advantages and efficiency of the present method. 


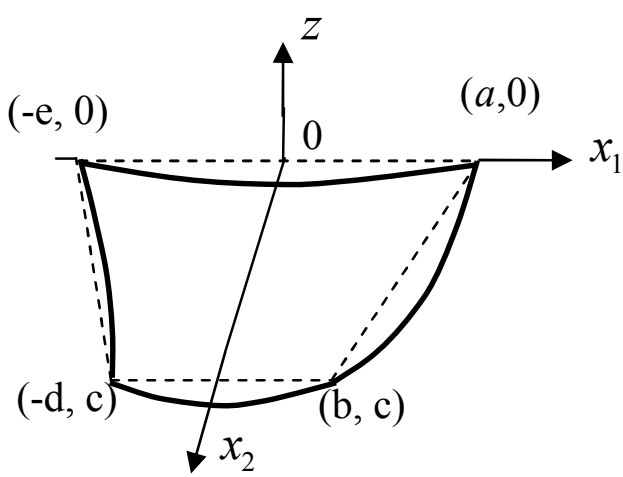

Fig.1 Slip clamped shallow spherical shell

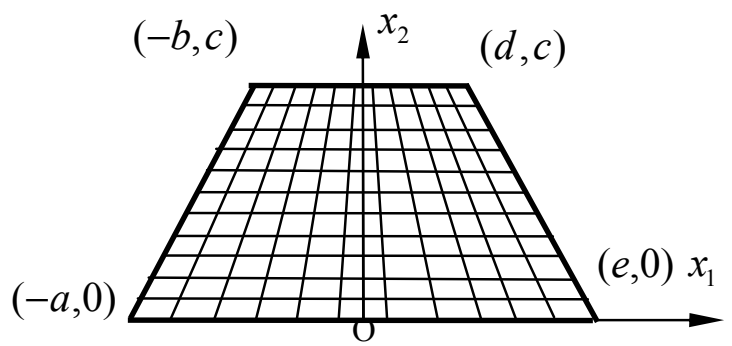

Fig.2 Division of trapezoidal integral domain

Tab.1 Natural frequency $f$ of slip clamped trapezoidal shallow spherical shell

\begin{tabular}{|c|c|c|c|c|c|c|}
\hline \multirow{3}{*}{$R$} & \multicolumn{6}{|c|}{ The mode rank } \\
\hline & \multicolumn{2}{|c|}{1} & \multicolumn{2}{|c|}{2} & \multicolumn{2}{|c|}{3} \\
\hline & RFT & FEM & $\overline{\mathrm{RFT}}$ & FEM & RFT & FEM \\
\hline 3 & 73.784 & 73.634 & 88.759 & 89.203 & 117.739 & 118.69 \\
\hline 5 & 68.930 & 68.773 & 84.766 & 85.235 & 114.76 & 115.73 \\
\hline 7 & 67.531 & 67.372 & 83.633 & 84.108 & 113.92 & 114.91 \\
\hline
\end{tabular}

\section{Conclusions}

In the present paper, the R-function theory is applied to study the free vibration of slip clamped trapezoidal shallow spherical shell on Winkler foundation. Compared with ANSYS finite element solution, it shows good agreement. R-function theory can also be used to effectively solve various boundary value problems in engineering by constructing a trial function that satisfies the boundary conditions and by combining with the method of weighted residuals such as the variational method and the spline-approximation [7-8].

\section{Acknowledgements}

The authors gratefully acknowledge the Natural Science Foundation of China (No. 11402099).

\section{References}

[1] V.L. Rvachev: Theory of R-function and Some of its Application (Nauk Dumka Press, Ukraine 1982) (In Russian).

[2] S.Q. Li and H. Yuan: Acta Mechanica Solida Sinica, Vol.25 (2012), p.37

[3] S.Q. Li and H. Yuan: Applied Mathematics and Mechanics (English Edtion), Vol.32 (2011),p.276

[4] H. Yuan, S.Q. Li and R.H. Liu: Applied Mathematics and Mechanics (English Edtion), Vol.28 (2007), p.847

[5] S.Q. Li and H. Yuan: Applied Mathematics and Mechanics (English Edtion), Vol.31(2010), p.635

[6] S.Q. Li and H. Yuan: Acta Mechanica Solida Sinica, Vol.23 (2010), p.370

[7] L. Kurpa, T. Shmatko and G. Timchenko: Composite Structures, Vol.93 (2010), p.225

[8] J. Awrejcewicz, L. Kurpa and A. Osetrov: ZAMM, Vol.91 (2011), p.458 